\title{
Infrared Moving Multi-target Tracking Based on Particle Filter and FCM
}

\author{
Hu Fanjun \\ Dept. of Aeronautical Ammunition Engineering \\ The First Aeronautical College of Air Force \\ Xinyang, China \\ e-mail: hufanjun@hotmail.com
}

\author{
Shi Wenjun \\ Dept. of Aeronautical Ammunition Engineering \\ The First Aeronautical College of Air Force \\ Xinyang, China \\ e-mail: shiwen_79_1@163.com
}

\begin{abstract}
An efficient approach based on particle filter and FCM is presented to realize moving infrared multi-target tracking under Island shore background. Some possible targets can be obtained and saved by processing IR data through denoising by median filter, extracting edge, identifying and eliminating sea-sky line, morphological filtering and etc. Data association and robust multi-target tracking can be realized by the proposed particle filter and FCM algorithm. The proposed approach is validated to track multi-target effectively by using actual infrared image sequences with Island shore background. Experiment results indicate the feasibility and effectiveness of the proposed method.
\end{abstract}

\section{Keywords- multi-target tracking; particle filter; FCM}

\section{INTRODUCTION}

The tracking and detection of infrared multi-target has been a key technology in the field of satellite early warning, precision guidance, warning and detection. Research on infrared multi-target tracking and detection under the Island shore background is nowadays one of the hot topics. Common methods of infrared multi-target are PDA(probabilistic data association) 、 JPDA(Joint probabilistic data association) and MHT(Multiple Hypothesis Tracking) [1], etc. The basic assumption of PDA algorithm is the mutual independence among targets, ignoring the interference of neighboring targets. It is only applicable to single target tracking; JPDA and MHT are regarded as the ideal method of multi-target tracking in complex environment, but the amount of calculation and storage quantity are large and it is hard for hardware realization [2]. PMHT (Probabilistic Multiple Hypothesis Tracking) algorithm can be influenced by tracking initialization, so it is not suitable for maneuvering targets.

According to the real-time and accuracy requirements of infrared multi-target tracking under Island shore background, based on particle filter, and also on the basis of preprocessing infrared image, an efficient approach based on particle filter and FCM is presented in this paper to realize moving infrared multi-target tracking under Island shore background. Aiming to eliminate false targets and track real target can be realized by preserving and comparing various targets position, gray, shape and moving characteristics during continuous tracking. The algorithm not only reduces the false alarm rate, but also ensures the amount of computation increasing linearly with the increase of the number of target.

\section{INFRARED IMAGE PREPROCESSING UNDER ISLAND SHORE BACKGROUND}

Typically, the Island shore background mostly appears near the sea-sky line region. Therefore, an approach is presented to eliminate Island shore background by detecting the sea-sky line, and then the influence of Island shore background during multi-target tracking can be removed. The flow chart of the extraction of image preprocessing is illustrated in figure 1 .

First, image edge extraction and segmentation can be realized through convolution operation with the Sobel operator.

Secondly, employ Hough transform to extract Sea-SkyLine by using the difference between sea surface and the sky. The significance of this procedure not only lies in reducing the range of recognition, but also in eliminating the interference of the target in the sky. Therefore, it is an effective processing method in target recognition. Because Sea-Sky-Line can be approximately seen as a line, Hough transform can be employed to realize the detection of the line. Please look at reference No.3 for the detail.

Finally, after edge detection of the image and eliminating treatment of Sea-Sky-Line, "closing” morphology operating can be used to process the image[4]. Finally, "8-connected" algorithm is applied to calculate the area, length to width ratio and centroid coordinate, then compare the result with empirical value and put $M$ targets and its parameters which are in accordance with the set condition into the storage sequence. Then data $\left\{T_{k}\left(d_{k}, a_{k}, g_{k}, s_{k}\right)\right\}$ ( $d_{k}$ : distance, $a_{k}$ : position, $g_{k}$ : average grey of the target region, $S_{k}$ : area) can be obtained.

\section{ALGORITHM IMPLEMENTATION FLOW}

$N$ candidate targets and its parameters can be obtained after preprocessing original IR. Continuous tracking of those targets can be realized by using the algorithm based on particle filter and FCM $[5,6]$. On the basis of the truth that particle filter algorithm is able to handle arbitrary nonlinear function and obey to non-Gaussian distribution, therefore, according to the target position and motion status of the current frame, particle filter algorithm can be used to estimate the position and motion state of the target in the next frame. Meanwhile, data of average grey and area of the 
target region in the current and next frames have been obtained by image preprocessing. Therefore, data association can be realized by particle filter and FCM. In this way, continuous and robust multi-target tracking can be realized, and the amount of computation is significantly reduced.

Rigid motion of the target can be described by a sixparameter affine transformation $A_{t}(\theta, S X, S Y, S X Y, T X, T Y)$ and a first-order Markov process modeling [7]. Here, $\theta$ : rotation angle, $S X, S Y$ : zoom scale, $T X, T Y$ : position coordinates.

The specific algorithm flow is as follows:

\section{A. Prior knowledge and particle state initialization}

Here, prior knowledge of target can be described by contour, and object contour can be obtained by fitting a number of curve control points, which is shown in Figure 2.

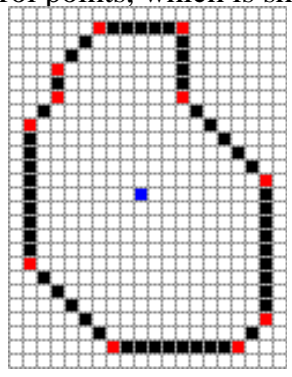

Figure 2. Curve control points.

Prior knowledge can be described by initial value of motion state at $k_{0}$ Moment.

$$
A^{\text {init }}=\left(\theta^{\text {init }}, S X^{\text {init }}, S Y^{\text {init }}, T X^{\text {init }}, T Y^{\text {init }}\right)
$$

Here, the number of particle is $N_{s}, \omega^{i}$ initial value is equal to 1 . Each particle represents a possible target state. In other words, each particle can be described by five parameters:

$$
\begin{gathered}
A^{i}=\left(\theta^{i}, S X^{i}, S Y^{i}, T X^{i}, T Y^{i}\right) \\
i=1,2, \cdots, N_{s}
\end{gathered}
$$

Correspondingly, the parameters $S^{i}$ of each particle in the shape space can be described as follows:

$$
\begin{aligned}
& S^{i}=\left(T X^{i}, T Y^{i}, S X^{i} \cos \theta^{i}-1\right. \\
& \left., S Y^{i} \cos \theta^{i}-1,-S Y^{i} \sin \theta^{i},-S X^{i} \sin \theta^{i}\right)
\end{aligned}
$$
as:

Initial value of each particle parameter can be assigned

$$
T X^{i}=T X^{\text {init }}+b_{1} \xi, T Y^{i}=T Y^{i n i t}+b_{2} \xi
$$

$$
\begin{gathered}
\theta^{i}=\theta^{i n i t}+b_{3} \xi, S X^{i}=S X^{i n i t}+b_{4} \xi \\
S Y^{i}=S Y^{i n i t}+b_{5} \xi
\end{gathered}
$$

Here, $\xi$ is a random number in $[-1,1], b_{1} 、 b_{2} 、 b_{3}$ 、 $b_{4}$ and $b_{5}$ is constant.

\section{B. Importance Sampling}

Predict

the affine parameters $A_{t}^{i}=\left(\theta^{i}, S X^{i}, S Y^{i}, T X^{i}, T Y^{i}\right)$, which describe the rigid motion of an object.

The specific flow is as follows:

At next $k_{t}(t>0)$ moment, predict each particle state by state transition equation.

Here, take the first order ARP equation:

$$
x_{t}=C x_{t-1}+D w_{t-1}
$$

As far as each particle is concerned:

$$
\begin{gathered}
T X_{t}^{i}=C_{1} T X_{t-1}^{i}+D_{1} w_{t-1}^{1}, \\
T Y_{t}^{i}=C_{2} T Y_{t-1}^{i}+D_{2} w_{t-1}^{2}, \\
\theta_{t}^{i}=C_{3} \theta_{t-1}^{i}+D_{3} w_{t-1}^{3}, \\
S X_{t}^{i}=C_{4} S X_{t-1}^{i}+D_{4} w_{t-1}^{4}, \\
S Y_{t}^{i}=C_{5} S Y_{t-1}^{i}+D_{5} w_{t-1}^{5} \\
i=1,2, \cdots, N_{s}
\end{gathered}
$$

Here, $\quad C_{1} \sim C_{5} \quad, \quad D_{1} \sim D_{5} \quad$ is constant, $w_{t-1}^{n}(n=1,2, \cdots, 5)$ is a random number in $[-1,1]$.

Each particle represents a possible motion state of the target, which is a possible contour of the target. All the particles represent all possible motion state.

\section{Particles weighted}

Compute particle weight and its iterative formula is as follows:

$$
\omega_{t}^{(i)}=\omega_{t-1}^{(i)} p\left(Z_{t} \mid X_{t}^{(i)}\right)
$$

Here, $p\left(Z_{t} \mid X_{t}^{(i)}\right)$ is likelihood function based on the observation model.

Typically, data association of target region during current and next frame can be realized by grey feature, structural feature and motion feature. Here, FCM is also involved in the algorithm. That is:

$$
\begin{aligned}
& p\left(Z_{t} \mid X_{t}^{(i)}\right) \propto \\
& \rho\left(T\left(d_{k-1}, a_{k-1}, g_{k-1}, s_{k-1}, r_{k-1}\right),\right. \\
& \left.T\left(d_{k}, a_{k}, g_{k}, s_{k}, r_{k}\right)\right)
\end{aligned}
$$




$$
\begin{gathered}
\rho=u_{d} *\left(\frac{d_{k}}{d_{k-1}}\right)^{2}+u_{a} *\left(\frac{a_{k}}{a_{k-1}}\right)^{2} \\
+u_{g} *\left(\frac{g_{k}}{g_{k-1}}\right)^{2}+u_{s} *\left(\frac{d_{s}}{d_{s-1}}\right)^{2}+u_{r} *\left(\frac{d_{r}}{d_{r-1}}\right)^{2} \\
u_{d}+u_{a}+u_{g}+u_{s}+u_{r}=1
\end{gathered}
$$

Here,

$\rho\left(T\left(d_{k-1}, a_{k-1}, g_{k-1}, s_{k-1}, r_{k-1}\right), T\left(d_{k}, a_{k}, g_{k}, s_{k}, r_{k}\right)\right)$ is the defined function of FCM. The size of the value indicates the degree of data association during current and next frame, when $\rho$ is larger, output value of FCM is higher. Here, according a great amount of experiments, we take $u_{d}=0.1, u_{a}=0.1, u_{g}=0.6, u_{s}=0.15$, $u_{r}=0.05$.

\section{ReSampling}

$$
N_{\text {eff }}=\frac{1}{\sum_{i=1}^{N}\left(\hat{\omega}_{k}^{i}\right)^{2}}
$$

If $N_{\text {eff }} \leq N_{\text {th }}$, particles with higher value will be resampled. Then turn to step 2 , reducing $w_{t-1}^{n}$ value range is to ensure resampled particle parameters are closer to the shape and position of target in current frame. Otherwise, turn to next step.

\section{E. Iteration}

Turn to next moment, contour of target in this frame can be obtained, which can be taken as the initial state and prior knowledge in next frame. Then turn to step 2.

Here, all possible target position can be obtained by affine transformation of the contour regions. Compare those contour regions with target contour in next frame. Through iteration, weighted and resampling, affine transform parameters can be finally fixed. In this way, continuous multi-target tracking can be realized.

\section{EXPERIMENT AND RESULT ANALYSIS}

Use infrared imaging device with a resolution of $320 * 240$, a rate of 50 frames/ second and responding wave band ranges from 8-14um to conduct infrared experiments and algorithm evaluation.

Experiment content is continuous tracking three ships under Island shore background (as shown in Figure 3). During the whole experiment, target 1 is moving to the left, target 3 is moving quickly to the right, and target 2 is slowly turning around. Figure 3, 5, 7 respectively is 10th, 356th and 750th frame original infrared image, and figure 4, 6, 8 respectively is 10th, 356th and 750th frame multi-target tracking image.

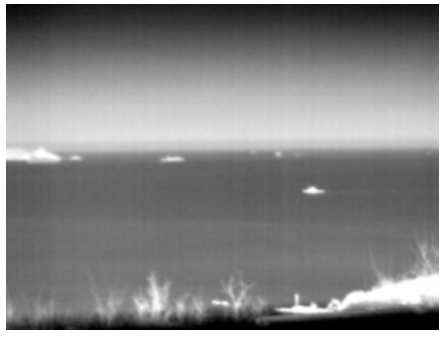

Figure 3. 10th IR raw image

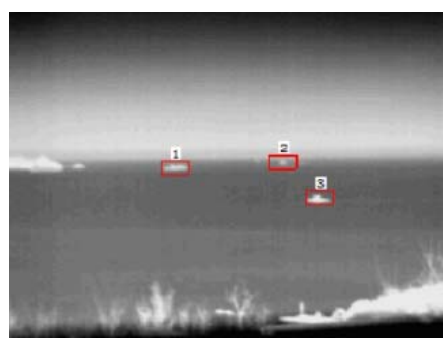

Figure 4. 10th IR processed image

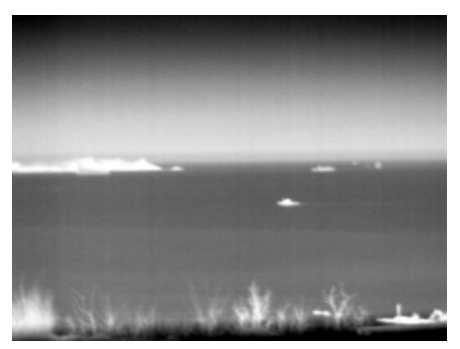

Figure 5. 356th IR raw image

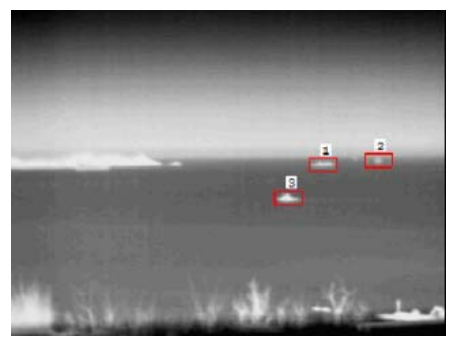

Figure 6. 356th IR processed image

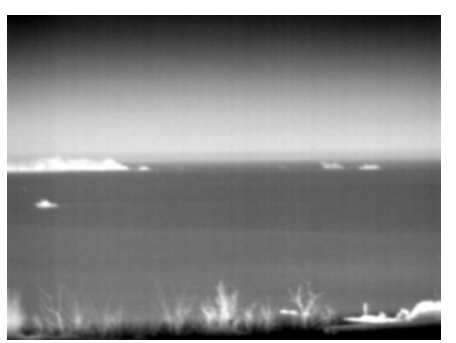

Figure 7. 750th IR raw image 


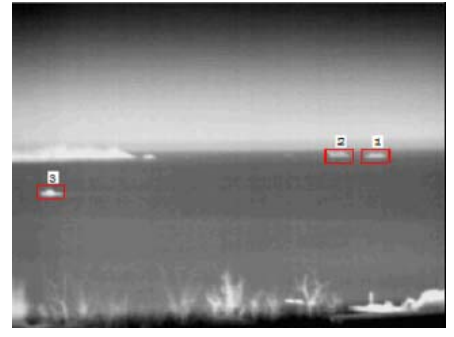

Figure 8. 750th IR processed image

Applying 2.3 algorithm flows, specific experimental procedures are as follows:

\section{Initialization}

Curve contour of target and its corresponding curve control points during current and next frame can be obtained by edge detection.

\section{Importance sampling}

Possible target contour can be predicted by curve control points' affine transformation. In other words, selecting 300 particles for each target represents target's region central point position. Then selecting particles for each target represents zoom scale parameters in affine transformation.

\section{Particles weighted}

Data association and particles weighted can be realized by comparing curve control points and region features in current frame with those obtained by the formula (8), (9), (10).

\section{Resampling}

Rule of iteration and termination in particle filter is as follows: when more than $95 \%$ of the whole particles are satisfied with the likelihood function $p\left(Z_{t} \mid X_{t}^{(i)}\right)<0.96$, iteration is finished. Then affine transformation parameters can be determined by the above particles. Otherwise, resample those particles with higher weight value. In other words, the resampling process cannot be terminated until all the affine transformation parameters are determined.
The experimental results indicate that the algorithm proposed by the paper can ensure robust and continuous moving infrared multi-target tracking under Island shore background.

\section{CONCLUSIONS}

In the whole processing, firstly Island shore background suppression can be realized by extracting Sea-Sky-Line and morphological filter, and then data association can be realized by particle filter and FCM, finally the purpose of continuous multi-target tracking can be achieved. The experimental results indicate that the iteration number in the algorithm is about 8 10 times, which is satisfied with realtime requirements. It is also easy for hardware implementation. Therefore, it is an ideal approach for infrared multi-target tracking under Island shore background. Certainly how to solve the problem of local deformation and occlusion during multi-target tracking are the research contents in the future.

\section{REFERENCES}

[1] G.W. Pulford, "Taxonomy of Multiple Target Tracking Methods," Proceedings of IEEE Conference on Radar Sonar Navigation, vol. 152,2005, pp. 291-304.

[2] HAO Xiao-ran, ZHANG You-zhi, "Method of Moving Point Target Detection in Image Sequence,” Infrared and Laser Engineering, 1999, pp.7-86.

[3] O Chutatape, L Guo, "A Modified Hough Transform for Line Detection and Its Performance,” Pattern Recognition, vol.32, 1999, pp.181-192.

[4] BRAGA-NETO U, CHOUDHARY M, Goutsias J, "Automatic Target Detection and Tracking in Forward-looking Infrared Image Sequences Using Morphological Connected Operators," Journal of Electronic Imaging, vol.13, 2004, pp. 802-813.

[5] DONG Chun-li, DONG Yu-ning, Liu Jie, “Object Tracking Algorithm Based on Particle Filtering and GVF-Snake," Chinese Journal of Scientific Instrument, vol.30, 2009, pp. 828-833.

[6] Selim S Z, Ismail M A, “K-means Type Algorithm," IEEE Transactions on Pattern Analysis and Machine Intelligence, vol.6, 1994, pp. 81-87.

[7] QIU Li-mei, HU Bu-fa, "3D Face Pose Estimation Based on Affine Transformation and Linear Regression,” Journal of Computer Applications, vol.26, 2006, pp. 2877-2883.

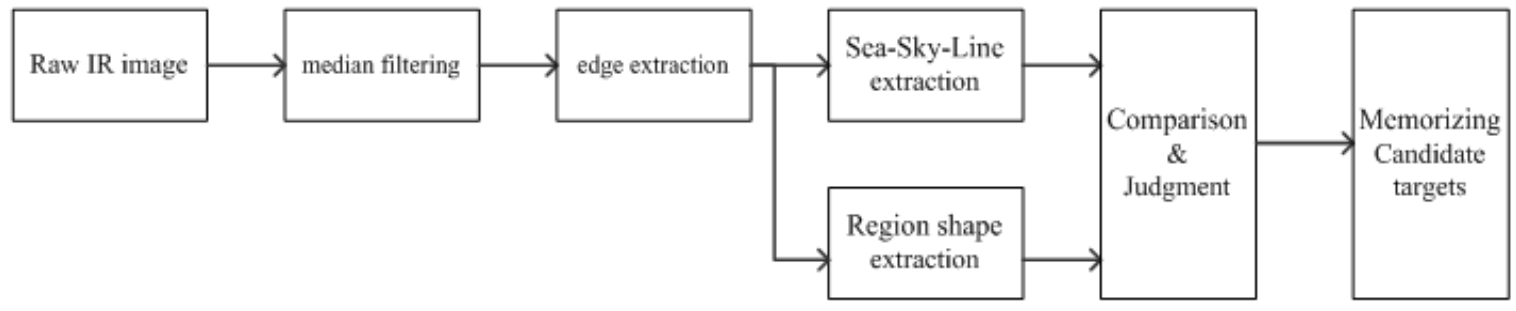

Figure 1. Flow chart of IR features extraction 